\title{
Left Ventricular Pseudoaneurysm Repair: A Case Report
}

\author{
Daniel Rothstein ${ }^{1}$, Michio Kajitani ${ }^{1}$, Mir Wasif $\mathrm{Ali}^{1}$, and Nahidh Hasaniya ${ }^{1}$ \\ ${ }^{1}$ St. Bernardine Medical Center
}

November 12, 2020

\begin{abstract}
A 50 year old male with a history of myocardial infarction 3 months prior presents to the emergency department with shortness of breath. Diagnostic workup revealed a left ventricular pseudoaneurysm of the inferolateral wall. Coronary artery disease and mitral valve regurgitation were concomitantly identified. Surgical intervention on these issues was successful. This case report highlights a rare but important complication of myocardial infarction.
\end{abstract}

Left Ventricular Pseudoaneurysm Repair: A Case Report

Authors: Daniel Rothstein, PA-C, Michio Kajitani, MD, PhD, Mir Wasif Ali, MD, FACS, Nahidh Hasaniya, $\mathrm{MD}, \mathrm{PhD}$

Dignity Health Medical Foundation Inland Empire Cardiothoracic Surgery Dept

San Bernardino, CA

Article word count: 1193

Keywords: Pseudoaneurysm, ventricular wall rupture, myocardial infarction

Corresponding author: Daniel Rothstein (415) 254-9931, d.rothstein0422@gmail.com

Data availability: N/A as no new data were created or analyzed in this study

Funding statement: none

Conflict of Interest: none

IRB Approval: N/A

Abstract

A 50 year old male with a history of myocardial infarction 3 months prior presents to the emergency department with shortness of breath. Diagnostic workup revealed a left ventricular pseudoaneurysm of the inferolateral wall. Coronary artery disease and mitral valve regurgitation were concomitantly identified. Surgical intervention on these issues was successful. This case report highlights a rare but important complication of myocardial infarction.

Introduction

Left ventricular pseudoaneurysm is a rare but challenging complication following myocardial infarction (MI). This occurs when the ventricular wall ruptures and the bleeding is contained by an adherent pericardium, forming a contained cavity. This prevents uncontrolled bleeding into the pericardium and subsequent tamponade. It has been reported in roughly $4 \%$ of MI patients and accounts for $23 \%$ of deaths related to $\mathrm{MI}^{1}$, however, it is possible that many cases go undetected because the initial presentation is sudden death. This 
type of complication is associated with a high likelihood of rupture and death, necessitating urgent surgical repair. Approximately 30-45\% of pseudoaneurysms will rupture. ${ }^{2}$ Pseudoaneurysm cavity walls do not contain any myocardium. In contrast, true ventricular aneurysms, which may also occur as a complication of MI, have a wide mouth and outpouching of thinned ventricular wall $(<5 \mathrm{~mm})$ that still contain remnants of myocardial tissue ${ }^{2}$. Both types of aneurysms can be accompanied by signs and symptoms of congestive heart failure (CHF), angina, and dyspnea. Distinguishing between the two diagnoses can be difficult. Patients can have ST-segment changes on Electrocardiography (ECG) and enlarged cardiac silhouette on chest x-ray. Physical exam may reveal a to-and-fro murmur, pericardial friction rub, or muffled heart sounds. A new systolic murmur may be present in $70 \%$ of patients. ${ }^{3}$ The diagnosis and structural changes to the LV are confirmed by angiography and echocardiography.

Case Report

A 50-year old male with a history of inferolateral MI three months prior to admission, presented to the Emergency Department with shortness of breath, orthopnea, and associated cough. Past medical history includes CHF (EF 30-35\%), schizophrenia, alcohol abuse, and polysubstance abuse. He was not on any medications prior to his acute MI. The patient is a difficult historian due to his psychiatric and substance abuse history, but he reports compliance with his medications.

Physical exam showed jugular venous distention, bilateral lower extremity $2+$ pitting edema, bibasilar crackles and a systolic murmur over the apex and left sternal border. All vital signs were stable. Chest x-ray showed an enlarged cardiac silhouette. Computed tomography (CT) scan revealed an outpouching of the left ventricle with a distinctly separate cavity (figure 1). Echocardiogram showed a large pseudoaneurysm with a $2.4 \mathrm{~cm}$ defect in the inferolateral wall of the left ventricle and moderate to severe mitral regurgitation. Coronary angiogram revealed an occluded circumflex artery with extensive disease in the left anterior descending artery (LAD). The patient was referred for surgical management.

Operative procedure

The patient underwent left ventricular pseudoaneurysm repair, coronary artery bypass grafting connecting the left internal mammary artery to the left anterior descending coronary artery, and a mitral valve replacement with 27-mm Medtronic Mosaic ${ }^{\mathrm{TM}}$ porcine tissue valve. Primary cannulation technique was standard central cannulation with a single venous cannula. Femoral cannulation was prepared as a backup in case of uncontrolled bleeding during pericardial dissection. A femoral arterial line was placed for accurate blood pressure monitoring and potential for intra-aortic balloon pump or percutaneous cannulation. A midline sternotomy was performed followed by careful dissection of pericardial adhesions using electrocautery and scissors. The patient was cooled down to $34^{\circ} \mathrm{C}$ after adequate heparinzation and the aorta was crossclamped. Cold blood cardioplegia was given antegrade and retrograde. This was repeated every 20 minutes throughout the procedure. Dissection was continued to delineate the pseudoaneurysm cavity on the posterolateral wall (figure 2). The pseudoaneurysm was entered and the defect repaired using a 2-0 Prolene ${ }^{\text {TM }}$ running suture reinforced with strips of Teflon ${ }^{\mathrm{TM}}$ felt on each side (figure 3 ).

Due to dense scarring that encased the multiple vessels, the LAD was the only feasible target for bypass. Before completing the bypass, a left atriotomy was made in order to examine the mitral valve. Upon visual inspection and direct water injection, it was thought that the mitral valve had become competent. The LAD graft was completed, the left atriotomy was closed, and the patient was weaned off cardiopulmonary bypass. The heart started beating in normal sinus rhythm. Transesophageal echocardiography (TEE) showed persistent moderate mitral regurgitation with tethering of the posterior leaflet, creating a large eccentric jet. The cross clamp was re-applied, cardioplegia was administered in the same manner, and the left atriotomy was re-opened. The mitral valve was then replaced using a 27-mm Medtronic Mosaic porcine tissue valve. Repeat TEE after coming back off bypass showed a competent valve with no paravalvular leak.

Dissection of the pericardial adhesions created extensive bleeding from the epicardial surface. Several topical hemostatic agents and blood products were used to control the bleeding prior to closing the chest. After adequate hemostasis, the chest was closed in the standard fashion and the patient was transferred to the 
ICU in stable condition.

The patient was discharged home on post-operative day nine in stable condition. He was evaluation in the clinic on post-operative day 23 and was doing well.

Discussion

Distinguishing between a left ventricular pseudoaneurysm and a true aneurysm is important in determining the need for surgical intervention. True aneurysms do not always require surgical repair and can be treated medically, whereas pseudoaneurysms require surgical repair due to a high risk of rupture. The timing of surgical intervention is important to improve patient outcomes. Current literature suggests that patients with post-infarct ventricular rupture, that immediate surgical intervention only be performed in the setting of hemodynamic instability. ${ }^{4}$ If surgery can be delayed through medical management or intra-aortic balloon pump, patients have consistently better survival rates. The timing of this delay is not well established and varied from as little as $>3$ days to as long as $>36$ days. Early surgery relative to time of infarct is associated with an in-hospital mortality rate of $52.4 \%$, whereas delayed surgery is associated with an in-hospital mortality rate of $7.6 \%{ }^{4}$. This is attributed to the maturation of the tissue surrounding the defect. The delay allows for myocardial fibrosis to occur and creates more favorable tissue for surgical repair. Angiography may be able to distinguish true aneurysms from false aneurysms as well as, identify the need for concomitant bypass grafting. Cardiac MRI with the use of gadolinium is the ideal imaging modality to able to provide a definitive diagnosis. ${ }^{2}$

Follow up data on LV pseudoaneurysm patients is limited, however, an analysis reported by Frances et al. showed that 25 patients (23\%) died at a median of three days postoperatively, but the surviving patients survived to a median of 46 weeks. Of the patients that were treated conservatively, 15 (48\%) died at a median of less than one week. ${ }^{3}$

The strategy to treat the accompanying mitral valve regurgitation can be complicated. It is very tempting to repair or preserve the native mitral valve, especially in younger patients, as was the case here. Ischemic mitral valve regurgitation is notorious for less than optimal outcomes when treatment by repair is attempted instead of replacement. In a complicated case like this, we recommend early decision making to proceed with replacement. This will shorten operative time and reduce the risk of other complications related to prolonged cardiopulmonary bypass time, such as excessive bleeding due to coagulopathy and multi system organ failure, which can lead to prolonged hospital stay and even death.

Conclusions

The survival of patients with pseudoaneurysms is low unless diagnosed early and repaired surgically.

References

1. Zoffoli G, Mangino D, Venturini A, et al. Diagnosing left ventricular aneurysm from pseudoaneurysm: a case report and a review in literature. Journal of cardiothoracic surgery. https://www.ncbi.nlm.nih.gov/pmc/articles/PMC2654444/. Published February 24, 2009. Accessed March 9, 2020.

2. Bisoyi S, Dash AK, Nayak D, Sahoo S, Mohapatra R. Left ventricular pseudoaneurysm versus aneurysm a diagnosis dilemma. Annals of cardiac anesthesia. https://www.ncbi.nlm.nih.gov/pmc/articles/PMC4900369/. Published 2016. Accessed March 9, 2020.

3. Frances C, Romero A, Grady D. Left ventricular pseudoaneurysm. Journal of the American College of Cardiology. https://www.ncbi.nlm.nih.gov/pubmed/9741493/. Published September 1998. Accessed March 9, 2020.

4. Papalexopoulou N, Young CP, Attia RQ. What is the best timing of surgery in patients with post-infarct ventricular septal rupture? Interactive cardiovascular and thoracic surgery. https://www.ncbi.nlm.nih.gov/pmc/articles/PMC3548527/. Published February 2013. Accessed March 21, 2020. 
5. Mackenzie JW, Lemole GM. Pseudoaneurysm of theLeft Ventricle. Texas Heart Institute Journal. 1994;21(4):296-301. https://www.ncbi.nlm.nih.gov/pmc/articles/PMC325192/pdf/thij000390058.pdf. Accessed March 8, 2020.

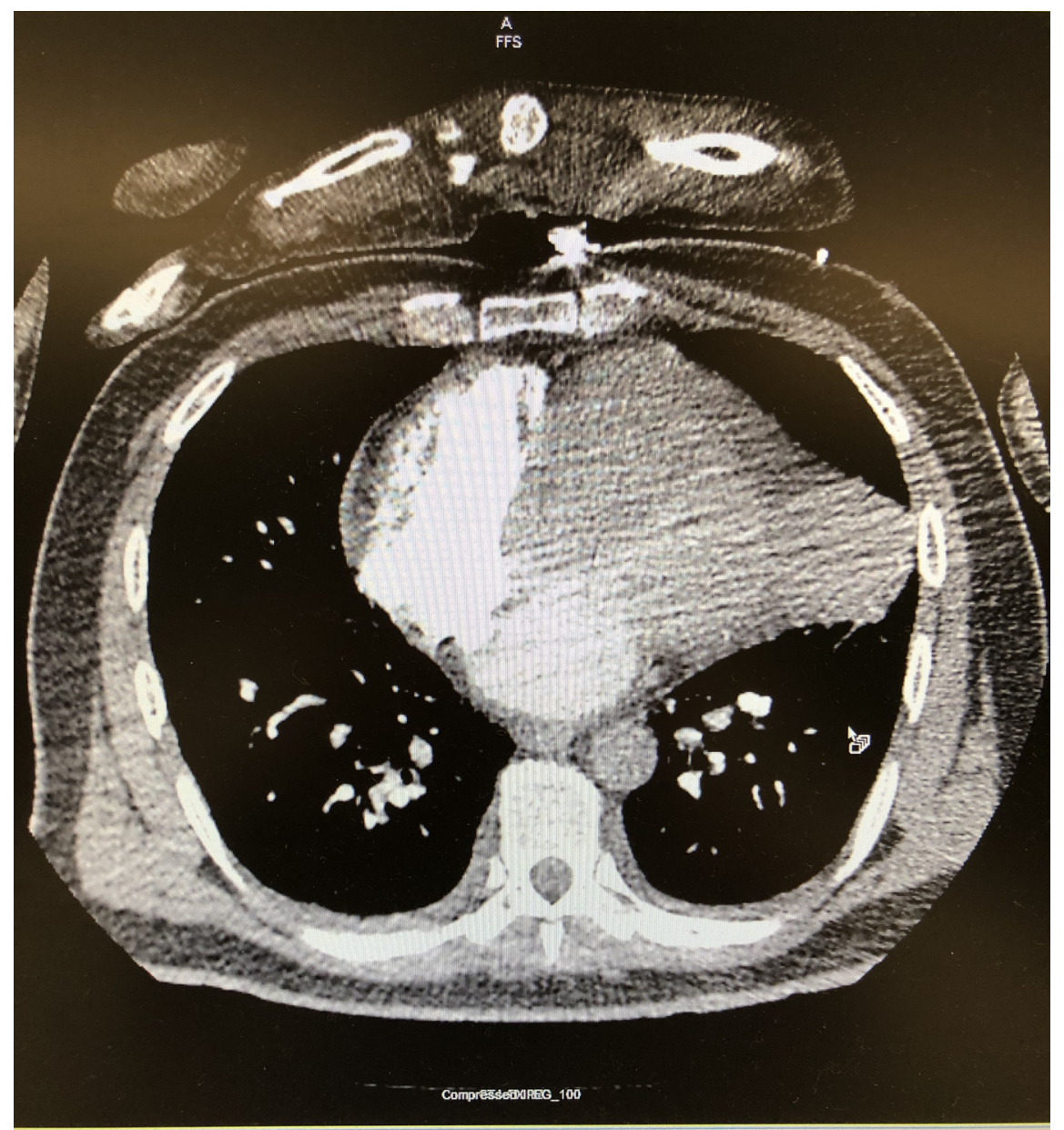




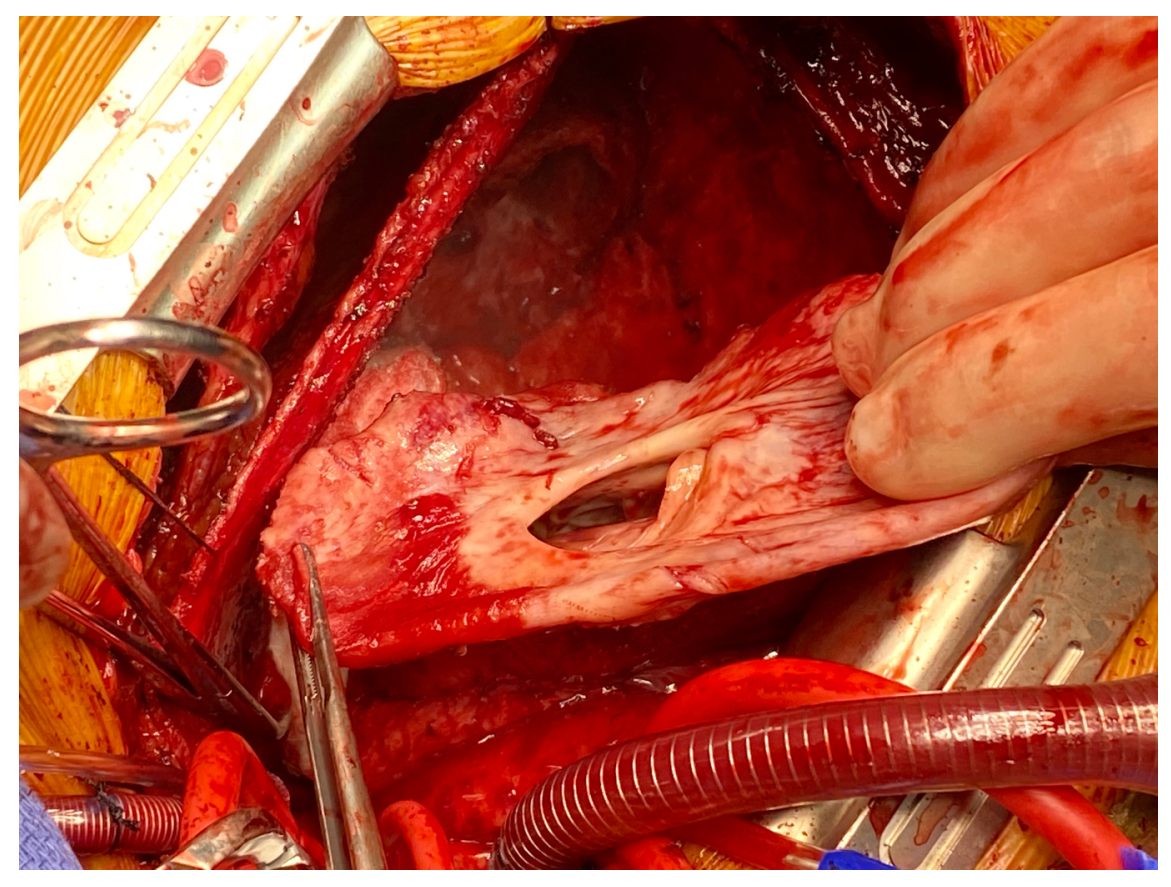




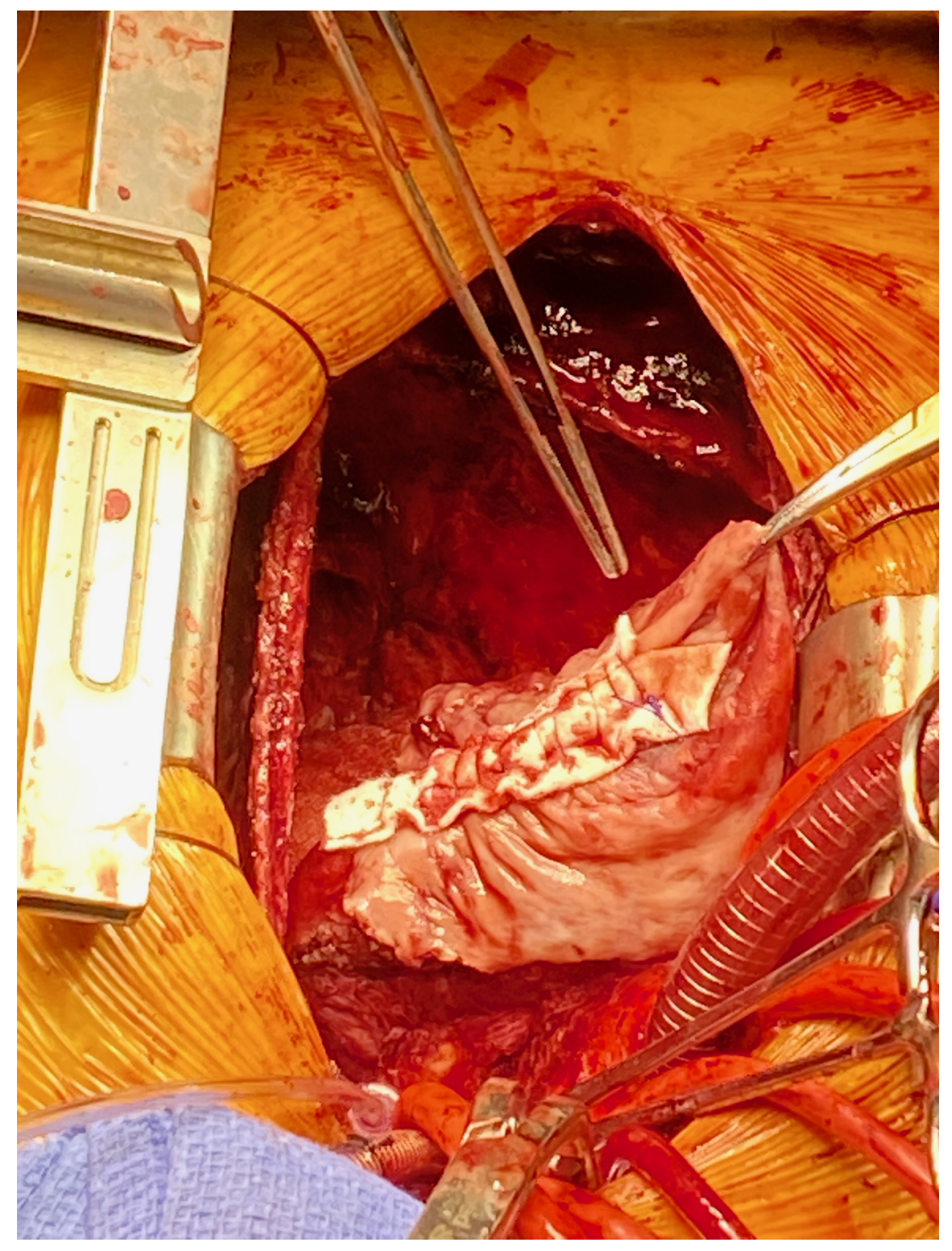

